CERN-TH.7224/94

IAS $94 / 24$

\title{
GAUGE FIELD IMPROVEMENT, FORM-SCALAR DUALITY, CONFORMAL INVARIANCE AND QUASI-LOCALITY
}

\author{
S. Deser円 \\ Theoretical Physics Division, CERN, Geneva, Switzerland \\ and \\ School of Natural Sciences, Institute for Advanced Study \\ Princeton, NJ 08540, U.S.A. \\ and \\ A. Schwimmer \\ Weizmann Institute, Rehovot, Israel and SISSA and INFN, Trieste,Italy
}

\begin{abstract}
The problem of maintaining scale and conformal invariance in Maxwell and general $N$-form gauge theories away from their critical dimension $d_{c}=2(N+1)$ is analyzed. We first exhibit the underlying group-theoretical clash between locality, gauge, Lorentz and conformal invariance requirements. "Improved" - traceless - stress tensors are then constructed; each violates one of the above criteria. However, when $d=N+2$, there is a duality equivalence between $N$-form models and massless scalars. Here we show that $N$-form conformal invariance is not lost, by constructing a quasilocal gauge invariant improved stress tensor. The correlators of the scalar theory are then reproduced, including the latter's trace anomaly. This resolves the old conformal invariance "paradox" between the dual partners.
\end{abstract}

CERN-TH.7224/94

April 1994

\footnotetext{
${ }^{1}$ On leave from Physics Dept., Brandeis Univ., Waltham, MA 02254, U.S.A.
} 



\section{Introduction}

Massless spin-1/2 fields have vanishing classical stress tensor trace in any dimension, while scalars can be "improved" to achieve $T_{\alpha}^{\alpha}=0$, thereby guaranteeing invariance under the special conformal (or full Weyl) group, in accord with their scale-independence. This is no longer the case for the Maxwell and general $N$-form gauge fields' stress tensor traces: they only vanish at critical dimension $d_{c}=2(N+1)$. It is therefore instructive to analyse the obstacles to improvement away from $d_{c}$. We shall first give a simple general group theoretical argument exhibiting the clash between gauge and conformal transformations. This argument involves the sensible assumptions of locality and Lorentz invariance. We will then construct explicit traceless stress tensors away from $d_{c}$, and see that they indeed violate gauge or Lorentz invariance. [Nonlocal improvement is trivial to accomplish, of course.]

A particularly interesting dimension is $d=N+2$ where there is a duality equivalence between $N$-forms and scalars. Here there is a longstanding apparent paradox: the scalar's stress tensor can of course be improved, whereas we have shown that the $N$-form's cannot. The resolution lies in the presence of a quasilocal (local on-shell) improvement for the latter that leads to equivalence of correlation functions in both languages, including in particular equality of trace anomalies of these - equally conformal invariant - dual models.

Most of our discussion will take place in the framework of free theories in flat space, the issue being preservation of the full conformal group. Equivalently, one could work in an external gravitational field where this translates to preservation of the Weyl group. We shall not be concerned here with delicate issues of zero-modes, addressing the main question of topologically trivial spaces.

\section{Group Theory}

A massless Lorentz invariant field theory is invariant under scale transformations

$$
x^{\mu^{\prime}}=\lambda x^{\mu},
$$

the basic field of the theory $\varphi_{j}(x)$ transforming as

$$
\varphi_{j}^{\prime}\left(x^{\prime}\right)=\lambda^{-\eta} \varphi_{j}(x)
$$

where $\eta$ is the dimension of the field, whose Lorentz properties are encoded in the index $j$. Correspondingly, there is a conserved dilatation current $D^{\mu}$ and charge $D=\int d^{d-1} x D^{0}$. If $D^{\mu}$ can be written in terms of a symmetric conserved (stress) tensor as

$$
D^{\mu}=x^{\nu} T_{\nu}^{\mu}
$$

then conservation of $D^{\mu}$ implies

$$
T_{\mu}^{\mu}=0
$$


The conformal currents $K^{\mu(\alpha)}$,

$$
K^{\mu(\alpha)}=\left(x^{2} g^{\alpha \nu}-2 x^{\nu} x^{\alpha}\right) T_{\nu}^{\mu}
$$

are then also conserved; the charges $K^{\alpha}$ generate the special conformal transformations

$$
x^{\mu^{\prime}}=\frac{x^{\mu}+b^{\mu} x^{2}}{1+2 b x+b^{2} x^{2}}
$$

on $\varphi_{j}$, corresponding to a symmetry of the theory. Now, since the special conformal transformations are generated by translations and the inversion

$$
x_{\mu}^{\prime}=\frac{x_{\mu}}{x^{2}},
$$

a necessary condition for the existence of a traceless energy momentum tensor is the invariance of the Lagrangian under (7). The transformation of the field under inversion is fixed by the conformal group to be

$$
\varphi_{j}^{\prime}\left(x^{\prime}\right)=\left(x^{2}\right)^{\eta} D_{j}^{k}\left(g^{\mu \nu}-2 x^{-2} x^{\mu} x^{\nu}\right) \varphi_{k}(x)
$$

where $D_{j}^{k}$ are the $\mathrm{SO}(\mathrm{d})$-Wigner functions in the appropriate representation evaluated for the $\mathrm{SO}(\mathrm{d})$ rotation in the argument of $D$ and $\eta$ is the scale dimension of (2).

Massless fields with vectorial or higher rank indices are described covariantly by actions which possess a gauge invariance. We limit ourselves here to systems described by bosonic $N$-forms $\varphi_{\mu_{1} \ldots \mu_{N}}$. [For recent general reviews of conformal invariance see [1] [ [4].] The gauge field action

$$
S=\int * d(\varphi *) d \varphi
$$

is invariant under gauge transformations defined by the $N-1$ form $\alpha$,

$$
\varphi \rightarrow \varphi+d \alpha .
$$

It is also invariant under dilations if we choose

$$
\eta=\frac{1}{2}(d-2),
$$

while dilations commute with the gauge transformation provided

$$
\eta_{\alpha}=\eta_{\varphi}-1
$$

As argued above, in order to have a traceless energy momentum tensor, the action (9) should be invariant under the transformation (7), (8) with $\eta$ given by (11). For if the action is invariant under the inversion (7) and the inversion does not commute with the gauge transformations, then (9) will be invariant also under a new gauge group generated by inversion times gauge transformation times inversion. So the gauge transformations (10) must commute with conformal transformations (7), (8). Now, the effect of diffeomorphisms $x^{\mu} \rightarrow x^{\mu^{\prime}}(x)$ is

$$
\varphi(x) d x \wedge \ldots \wedge d x=\varphi^{\prime}\left(x^{\prime}\right) d x^{\prime} \wedge \ldots \wedge d x^{\prime}
$$




$$
\alpha(x) d x \wedge \ldots \wedge d x=\alpha^{\prime}\left(x^{\prime}\right) d x^{\prime} \wedge \ldots d x^{\prime},
$$

which automatically commute with (10). The conformal group considered as a subgroup of diffeomorphisms will therefore commute with (10), but then the dimension $\eta$ is clearly fixed by (13) to be

$$
\eta_{\varphi}=N, \quad \eta_{\alpha}=N-1 .
$$

This requirement is equivalent to the form being inert under a Weyl transformation. Therefore the action for an $N$-form in $d$ dimensions will be invariant under the full conformal group momentum tensor iff

$$
d=d_{c}=2(N+1) .
$$

At $d_{c}$, the conformal charges will be gauge invariant automatically. The above arguments assumed implicitly that the gauge fields transform in the usual, local way under the conformal group. This assumption will be important in our analysis of $N$-form-scalar duality in Section 4 , to explain the apparent discrepancy in their conformal behaviour at $d=N+2$.

In light of the above general result, there can be no strictly local (but see Section IV), Lorentz and gauge invariant conserved traceless stress tensor away from $d_{c}$. Hence we have two options if we insist on improvement:

a) Work entirely in terms of the non-covariant but gauge-invariant variables. [Although gauge-fixing is not strictly necessary in this Abelian framework, one may think in terms of a physical "Coulomb" gauge choice here.] This will, however, entail loss of Lorentz invariance.

b) Choose a covariant (Landau or Feyman) gauge; the resulting generators will depend on unphysical (ghost) degrees of freedom, due to loss of gauge invariance.

\section{$3 \quad$ Explicit Improvement}

We now turn to explicit realizations of condition (田), $T_{\alpha}^{\alpha}=0$ for our systems. Recall that the possibility of improving a stress tensor rests on its non-uniqueness since it is not a physical local current in the absence of gravitational coupling; one may add to $T^{\mu \nu}$ any quantity of the form:

$$
\Delta^{\mu \nu}=\partial_{\alpha \beta}^{2} H^{\mu \alpha \nu \beta}, \quad H^{\mu \alpha \nu \beta}=H^{\nu \beta \mu \alpha}=-H^{\beta \nu \mu \alpha}
$$

since $\Delta^{\mu \nu}$ is identically conserved, symmetric and does not alter the Lorentz generators for arbitrary (aymptotically well-behaved) functions $H$ with the above symmetries. This is equivalent to adding non-minimal gravitational couplings $\sim R_{\mu \alpha \nu \beta} H^{\mu \alpha \nu \beta}, R_{\mu \nu} H^{\mu \nu}$ or $R H$ to the original minimal action. Thus, our task is to find $\Delta^{\mu \nu}$ whose traces cancel those of the original $T^{\mu \nu}$, at least on shell. If this can be accomplished in a given gauge, the theory will have conserved dilatation and conformal currents (31), (5) [This is the basis of the scalar theory's improvement, using $\Delta_{\mu \nu}=\frac{1}{4}(d-2)(d-1)^{-1}\left(\eta_{\mu \nu} \square-\partial_{\mu \nu}^{2}\right) \phi^{2}$.] 
We begin with vector theory. Since the Maxwell tensor's trace is $(1-d / 4) F_{\mu \nu}^{2}, H$ would have to be bilinear in the vector potentials. In option a), we use the fact that as for any free Abelian gauge theory, we can express all quantities entirely in terms of the physical modes; here the transverse spatial components $\left(\mathbf{A}^{T}, \dot{\mathbf{A}}^{T}\right), \nabla \cdot \mathbf{A}^{T} \equiv 0$, due to the Gauss constraint. Dropping the " $T$ " notation henceforth, we write

$$
\begin{aligned}
T_{\alpha}^{\alpha} & =\left(1-\frac{d}{4}\right) F_{\mu \nu}^{2}=\frac{1}{2}(d-4)\left[\dot{\mathbf{A}}^{2}-\left(A_{i, j}\right)^{2}+\partial_{i j}^{2}\left(A_{i} A_{j}\right)\right] \\
& \cong \frac{1}{4}(d-4)\left[2 \partial_{\mu \nu}^{2}-\eta_{\mu \nu} \square\right]\left(A^{\mu} A^{\nu}\right), A_{\mu} \equiv\left(0, A_{i}\right) .
\end{aligned}
$$

The last equality is valid on shell $(\square \mathbf{A}=0)$ and the space-time notation is purely for convenience. It is easy to check that if we introduce

$$
\begin{aligned}
H^{\mu \alpha \nu \beta} & =a \eta^{\mu \nu} A^{\alpha} A^{\beta}+b \eta^{\mu \nu} \eta^{\alpha \beta} A^{2}+\text { symm. } \\
a & =\frac{(4-d)}{2(d-2)}, \quad b=\frac{d(d-4)}{4(d-1)(d-2)} .
\end{aligned}
$$

the corresponding $\Delta_{\mu}^{\mu}$ just cancels (17). The dilatation generator $D$ is likewise improved, to the desired form, $D=-\int \dot{\mathbf{A}}\left(\frac{d-2}{2}+r \cdot \nabla\right) \mathbf{A} d^{d-1} r$, as is $K^{0}$, which acquires a non-moment term $=\frac{d-2}{2} \int \mathbf{A}^{2} d^{d-1} r$; finally, $K^{i}$ is "improved" in that the part proportional to $-\int x^{i} \dot{\mathbf{A}} \cdot \mathbf{A}$ acquires an overall $(d-2)$ coefficient, exactly like the scalar field's improvement. [The special case $d=2$ of course corresponds to the trivial theory in which there is no $\mathbf{A}^{T}$ at all!] However, the various $\Delta^{\mu \nu}$ components are not Lorentz covariant and the improved generators have the "wrong" commutators with the (unaltered) Lorentz generators. This can be seen upon reintroducing the usual longitudinal and temporal components of $A_{\mu}$ through setting $A_{\mu} \rightarrow A_{\mu}+\partial_{\mu} \alpha$, thereby shifting the values of $\Delta_{\mu}^{0}$. This difficulty incidentally shows the limitation on the folklore that a free Abelian gauge theory can be entirely treated in terms of its transverse, physical, modes with Lorentz invariance always taking care of itself.

If we retain manifest Lorentz invariance, as in option b), keeping the usual $A_{\mu}$, then

$$
\begin{aligned}
T_{\alpha}^{\alpha} & =2\left(1-\frac{d}{4}\right) \partial_{\mu} A_{\nu}\left(\partial^{\mu} A^{\nu}-\partial^{\nu} A^{\mu}\right) \\
& \cong 2\left(1-\frac{d}{4}\right)\left[\left(\eta_{\mu \nu} \square-2 \partial_{\mu \nu}^{2}\right) A^{\mu} A^{\nu}+2 \partial_{\mu}\left(A^{\mu} \partial_{\nu} A^{\nu}\right)\right]
\end{aligned}
$$

The last term in (19) is a non-superpotential residue that cannot be cancelled without gaugefixing, in accordance with our general arguments. In Landau gauge the additional term vanishes, and $\Delta^{\mu \nu}$ can be chosen as in Eqs. (17), (18). In Feynman gauge the improved tensor will be simply given by d copies of the tensor for scalars, where the Lorentz index of the vector field acts like an internal symmetry index. If one adds the required FP ghosts one has a conformally invariant action, formally equivalent to the original one. The price here is, of course, that conformal inversion does not commute with the BRS operator. Therefore the symmetry does not act within the physical Hilbert space and so this improved tensor is not an allowed operator. 
There is a simple way, related to our group theoretical arguments, to see why scale invariance cannot be reinstated: the scale dimension of $A_{\mu}$, as obtained by acting on it with $D$, must be compatible with gauging $A_{\mu} \rightarrow A_{\mu}+\partial_{\mu} \alpha$; but $\alpha$, being non-dynamical, has scale dimension zero. Hence $A_{\mu}$ must have dimension unity, leading to $d_{c}=4$. The same problem holds for the antisymmetric tensor field away from $d=6$. The simple argument here is that the potential $B_{\mu \nu}$ is invariant under $B_{\mu \nu} \rightarrow B_{\mu \nu}+\left(\partial_{\mu} \lambda_{\nu}-\partial_{\nu} \lambda_{\mu}\right)$, i.e., under a transverse vector, $\lambda^{\mu} \epsilon^{\mu \nu} \ldots \partial_{\nu} \Lambda \ldots$, so that really $\delta B_{\mu \nu} \sim \partial^{2} \Lambda$ and it must have dimension 2 , compatible only with $d_{c}=6$.

For the antisymmetric tensor theory, whose Lagrangian is $-1 / 6 H_{\mu \nu \alpha}^{2}$, the stress tensor trace is

$$
T_{\alpha}^{\alpha}=\left(1-\frac{d}{6}\right) H_{\mu \nu \alpha}^{2} \quad H_{\mu \nu \alpha}=\partial_{[\alpha} B_{\mu \nu]} .
$$

the covariant from corresponding to $(\sqrt{19})$ is

$$
\begin{aligned}
T_{\alpha}^{\alpha} & =\frac{1}{2}(6-d) \partial_{\alpha}\left\{B_{\mu \nu}\left(\partial_{\alpha} B_{\mu \nu}+2 \partial_{\nu} B_{\alpha \mu}\right\}\right. \\
& \cong(6-d)\left[\frac{1}{4} \square B_{\mu \nu}^{2}+\partial_{\nu \alpha}^{2}\left(B_{\mu \nu} B_{\alpha \mu}\right)-\partial_{\alpha}\left(B_{\alpha \mu} \partial_{\nu} B_{\mu \nu}\right)\right]
\end{aligned}
$$

with a single derivative term vanishng only in Lorentz gauge $\partial_{\nu} B_{\mu \nu}=0$. In terms of the physical mode, $T_{\alpha}^{\alpha}$ is a double derivative, however. Here, the gauge invariant (but not Lorentz scalar) field is $\varphi$, where $B^{i j}=\epsilon^{i j k} \partial_{k} \varphi$, working in $d=4$ for simplicity. It follows by use of the constraint $\partial_{k} H^{0 i k}=0$ that $T_{\alpha}^{\alpha}$ is simply proportional to $\square \dot{\varphi}^{2}$ (or to $\square\left(\partial_{\mu} \varphi\right)^{2}$ plus a superpotential) so that one may use

$$
\Delta_{\mu \nu} \sim\left(\eta_{\mu \nu} \square-\partial_{\mu \nu}^{2}\right) \dot{\varphi}^{2}
$$

to cancel $T_{\alpha}^{\alpha}$. [This discussion and the final form (22) are actually valid for all dual models.] Covariantizing (22) by reinstating the gauge parts of $B_{\mu \nu}$, however, again reveals its gauge variance; one cannot have both symmetries simultaneously away from $d=6$. We shall return to this point below.

\section{Dualities}

It is well known, and leads to a well-known paradox (see for example [2]), that in $d=4$ the $N=2$ action is equivalent to that of a massless scalar, as is the Maxwell action in $d=3$ and more generally the $N$-form's action in $d=N+2$ where the dual field strength is a 1 -form ${ }^{*} H$. For, consider there the Lagrangian

$$
\mathcal{L}=-\frac{1}{2} A_{\mu}^{2}+{ }^{*} H^{\mu} A_{\mu} .
$$

Eliminating $A_{\mu}$ gives $\mathcal{L} \sim{ }^{*} H^{2}$, while varying with respect to the (Lagrange multiplier) $B_{\alpha \beta}$ field tells us that $A_{\mu}$ is the gradient of a scalar $\Psi$, and $\mathcal{L} \sim\left(\partial_{\mu} \Psi\right)^{2}$. The gauge-invariant stress tensor is

$$
T^{\mu \nu} \sim{ }^{*} H^{\mu *} H^{\nu}-\frac{1}{2} g^{\mu \nu}\left({ }^{*} H\right)^{2}, \quad T_{\alpha}^{\alpha} \sim-{ }^{*} H^{2} .
$$


It is conserved by virtue of the Bianchi identity $\partial_{\mu}{ }^{*} H^{\mu}=0$. The only way to improve it without losing gauge or Lorentz invariance would seem to be a non-local one. Indeed, we may postulate

$$
\Delta_{\mu \nu}=\frac{1}{3}\left(\eta_{\mu \nu} \square-\partial_{\mu \nu}^{2}\right) \square^{-1}\left({ }^{*} H\right)^{2} .
$$

This apparently unacceptable non-local operator has a saving feature, however: its matrix elements between zero mass gauge invariant states are local, the $\square^{-1}$ is cancelled. Since the only gauge invariant operators in the theory are $H^{*}$ and expressions built from them, the simplest way to verify this is to consider the Fourier transform of the correlator $C_{\mu \nu \alpha \beta}$ of $\Delta_{\mu \nu}(x)$ with $H_{\alpha}^{*}(y)$ and $H_{\beta}^{*}(z) . \Delta$ carries momentum $q$ and the $H^{*}$ s $k_{1}$ and $k_{2}$, respectively. Taking the discontinuity in $k_{1}$ and $k_{2}$ puts the states on mass shell and we obtain:

$$
\operatorname{disc} C_{\mu \nu \alpha \beta} \sim \delta\left(k_{1}^{2}\right) \delta\left(k_{2}^{2}\right) k_{1 \alpha} k_{2 \beta}\left(\eta_{\mu \nu} q^{2}-q_{\mu} q_{\nu}\right)
$$

using the gauge invariant propagator $\left\langle{ }^{*} H^{\alpha *} H^{\beta}\right\rangle \sim k^{-2}\left(k^{\alpha} k^{\beta}-g^{\alpha \beta} k^{2}\right)$. To go off mass shell, we simply complete (26) to its full analytic structure, i.e. the correlator is given by:

$$
C_{\mu \nu \alpha \beta} \sim\left(k_{1}^{2} k_{2}^{2}\right)^{-1} k_{1 \alpha} k_{2 \beta}\left(\eta_{\mu \nu} q^{2}-q_{\mu} q_{\nu}\right)
$$

Naive use of the gauge invariant propagator would add terms singular in $q^{2}$ and proportional to $k_{1}^{2}$ or $k_{2}^{2}$. Discarding these terms is completely consistent with analyticity, gauge invariance and Lorentz invariance, but not with the Bianchi identity off-shell, and it corresponds to the (required) use of the equation of motion $\left(k_{i}^{2}=0\right)$ in the scalar improvement.

Once the expression for the basic correlator (27) is given, calculating that of any number of stress tensors is straightforward, and the result is identical to that obtained in terms of the improved scalar: we are simply realizing the ${ }^{*} H^{\alpha} \sim \partial^{\alpha} \Psi$ correspondence in the Feynman diagrams. In particular, the trace anomalies calculated with the improved tensor in the scalar or $N$-form language automatically have the same values. Actually, since the anomaly depends only on mass-shell matrix elements [3], one can prove the equality starting from (26).

The improvement presented here has its counterpart in operator language, where we saw at Eq. (22) how in the physical sector (obeying the Gauss constraint) of Hilbert space, the improvement becomes local. We will discuss elsewhere the formulation of improvement in an external gravitational field and the realization of the Weyl group. We also remark that a similar treatment is possible in the trivial case of $d=N+1$ and the $N$-form is "dual to nothing". Here our procedure would simply yield a vanishing correlator of the improved stress tensor with any gauge invariant operator. Finally, we mention that in addition to the usual massless scalar action, there are two others that are scale or Weyl invariant at $d=4$, although they are less physical. Both have $N=2$ counterparts. We simply list them here. The first is $\mathcal{L}=-\frac{1}{2} \Psi \square^{2} \Psi$, which can be locally improved, but represents a dipole ghost excitation. Its counterpart is nonlocal. The other is $\mathcal{L}=\left[\left(\partial_{\mu} \Psi\right)^{2}\right]^{2}$; its $N=2$ partner is a non-polynomial action $\mathcal{L} \sim\left({ }^{*} H^{2}\right)^{2 / 3}$. Both actions are manifestly Weyl invariant, being homogeneous of degree zero in the metric. 


\section{Conclusion}

We have seen, by general arguments and by specific examples that improving $N$-form actions away from critical dimension required either non-locality or loss of Lorentz or gauge invariance. However, for dimension $d=N+2$ where the $N$-form is dual to a scalar, we saw that improvement can be carried out quasilocally (on-shell). This possibility provided the resolution of the ancient "paradox" of conformal non-invariance on the $N$-form side, and in particular explained the equality of the conformal anomalies of both partners.

This work was supported by NSF grants PHY-93-15811, 92-45316, by Israel-US Binational grant 89-00140, and by the Ambrose Monell Foundation.

\section{References}

[1] J. Polchinsky, Nucl. Phys. B303 (1988) 226.

[2] M.J. Duff, "20 Years of the Weyl Anomaly", Texas A\&M preprint CPT-TAMU/06-93, hep-th 9308075 preprint (1993);

M.T. Grisaru, N.K. Nielsen, W. Siegel and D. Zanon, Nucl. Phys. B247 (1984) 157.

[3] S. Deser, I. Halliday and A. Schwimmer, in preparation.

[4] H. Osborn and A. Petkos, preprint Cambridge DAMTP 93/31 (1993). 